\title{
In search of a new frontier in Astronomy
}

\section{Opinion}

I am trying to understand this universe as an Astrophysicist along with my students.

Most of the matter in the universe is non-luminous matter. According to the standard model of cosmology the Universe contains $4.9 \%$ luminous, $26.8 \%$ Dark matter and $68.3 \%$ dark energy. Neutrino as a dark matter played its role in the evolution of the universe. There are 3 types of Neutrinos. They are Electron Neutrino, Taon Neutrino, and Muon Neutrino. They now illuminated the field of High Energy Physics. In recent years Neutrino Astronomy has found its rightful place in cosmology. With the opening of X-ray and Gammaray astronomy we realized that some new Neutrino form might be essential to understand dark matter. There are galaxies which are supposed to be seeds of Dark matter. X-ray lines of definite energy have been observed in the galaxies supposed to be infested by dark matter naturally opinionated some form of Neutrinos not so far discovered may be responsible for X-ray energy in those dark matter galaxies. A new kind of Neutrino has been envisaged to explain these events. These are the Sterile Neutrinos which are mostly affected by gravity and may split into photons in a magnetic field by what is known as Casimir effect. Sterile Neutrino is a right handed neutrino, known as warm dark matter. Recently $3.5 \mathrm{keV}$ and $2.5 \mathrm{keV}$ lines are observed and it is explained as due to the decay of $7 \mathrm{keV}$ and $5 \mathrm{keV}$ sterile neutrinos. The dark matter search is on. This has opened up a complete new frontier of neutrinos. It is interesting to look for sterile neutrinos of different masses .It is a good attempt to explore many high energy events in terms of sterile neutrinos.

After 60 years of the seminal paper by Burbidge et al. (Review of Modern Physics, 1957, Vol 29) the subject of Nuclear Astrophysics is still invigorating a new pathways have been discovered. Many new features of elemental abundances have come up for scrutiny once again. In the branch of heavy element nucleosynthesis many new and exotic elements were predicted and discovered. It shows clearly that application of nuclear and high energy physics has elaborated the development of the subject in the laboratories to give a clue to discover new elements in the universe, which may be interesting for the studies of new exotic worlds in the form of exo-planets.

Even the hydrogen burning cycles which have been vital for
Volume I Issue 2 - 2017

\author{
Kalpana Duorah \\ Department of Physics, Gauhati University, India
}

Correspondence: Kalpana Duorah, Department of Physics, Gauhati University, Guwahati-78I0I4, India, Tel 036I-257053।, Email khowang56@yahoo.co.in

Received:September II, 2017 | Published: September 20, 2017

P-process element synthesis have been found to be reasonably good in predicting certain elements like Aluminium 26 etc. which were otherwise not accessible to usual nucleosynthesic processes. This is good because that work is giving us in all frontiers to understand all the elements in the Periodic table and beyond.

Another very interesting event in the universe in the Gamma-ray burster. They are believed to be associated with neutron stars and their associated magnetic fields. They are Magnetars. These Magnetar phenomenons may play a very crucial role in these kinds of high energy events.

Therefore it will be interesting to prove deeply the whole of neutron stars, Gamma-ray busters and Magnetars in a coherent way.

In the 20's of this century will open up new frontiers in observational Astronomy via LSST. XMM NEWTON, LAT. It will be interesting how the population on Earth is going to participate in these events in a variegated ways.

The scientific world will be enormously enriched with these kinds of activities and the humanity will be benefited in the new knowledge age.

\section{Acknowledgments}

None.

\section{Conflicts of interest}

The author declares there is no conflict of interest. 